\title{
An IR-UWB multi-sensor approach for collision avoidance in indoor environments
}

\author{
Faheem Khan, Member, IEEE, Stéphane Azou, Senior Member, IEEE, Roua Youssef, Member, IEEE Pascal \\ Morel, Emanuel Radoi, Senior Member, IEEE, and Octavia A. Dobre, Fellow, IEEE
}

\begin{abstract}
This paper aims to propose new techniques to detect and distinguish humans from moving machines in indoor environments. Although many research efforts have been already dedicated to humans' indoor detection, most of the work has been focused on counting people and crowd measurement for consumer business applications. Our objective is to develop a reliable approach for humans' indoor detection and localization aiming at avoiding collisions inside a mixed Industry 4.0 manned and unmanned environment, so that to enhance the personal and equipment safety and to prevent unwanted intrusions. An original aspect of our research is that we have worked on the real time estimation of humans' and moving machines' positions, while addressing the problems of multipath components and noise clutter detection. A multi-pulse constant false alarm rate detection algorithm is also proposed for removing the misdetections due to heavy clutter components in the indoor environment. Four impulse radio ultrawideband transceivers are placed in a specific geometry and data fusion is performed to reduce the influence of multipath and noise on the detection process. A convolutional neural network (CNN) is then used to extract the patterns corresponding to a moving machine and humans and classify them accordingly. Experiments have been carried out in two different indoor environments to demonstrate the performance of the proposed algorithms.
\end{abstract}

Index Terms-Impulse radio ultrawideband, human detection and localization, multi-path, mono-static radar array, smart sensing, collision avoidance.

\section{INTRODUCTION}

$\mathbf{H}$ Uman detection and localization is currently a hot research topic due to its real world applications such as safety, energy management, collision detection and asset tracking. Recently, a variety of technologies have been proposed for indoor human detection such as passive infrared (PIR) [1], LIDAR [2], WiFi [3] and optical camera [4]. However, these technologies have challenges associated with inaccuracy, environmental robustness and system complexity [5]. In addition, camera-based tracking requires clear view of the target and right lighting to work satisfactorily. These drawbacks explain the increasing interest in radar-based human detection. Differently from some recently reported research [6]-[8], where millimeter wave radar has been used for object localization and tracking, in this paper we have chosen to work with ultrawideband (UWB) radar sensors, due to their high

Faheem Khan, Roua Youssef and Emanuel Radoi are with the University of Brest, Lab-STICC, CNRS, UMR 6285, F-29200 Brest, France (e-mail: \{fkhan, ryoussef, radoi\}@ univ-brest.fr).

Stéphane Azou and Pascal Morel are with ENIB, Lab-STICC, CNRS, UMR 6285, F-29200 Brest, France (e-mail: \{stephane.azou, pascal.morel\}@enib.fr).

Octavia A. Dobre is with Memorial University of Newfoundland, Canada (e-mail: odobre@mun.ca). resolution characteristic [9]-[12] and the fact that, unlike the camera sensors, there are no related privacy concerns. The low cost of UWB sensors is also an advantage compared to alternative approaches using cameras or millimeter wave radars [13]. Moreover, UWB sensors provide better object penetration [14]-[16] compared to narrowband radar systems, require low transmission power and are not subject to interference issues [17]-[24]. Our work is related to indoor collision avoidance system (ICAS) for humans, automatic guided moving machine or free roaming machines inside a smart factory-like environment [25]-[27]. Since the collision avoidance technologies mainly depend on the distance of obstacle from the human [28], [29], it is very important to accurately localize the targets inside the indoor environment. The precise localization of moving machines and humans may reduce the cost associated with automatic guided machines damage due to collision and ensure the safety of employees working alongside the industrial machines. In our proposed set-up, fixed UWB anchors will determine the position of each moving object in the indoor environment and if there is a risk of collision, then it will broadcast the navigation information to the automated moving machine or to its operator in order to avoid this event. Although there are many benefits of using UWB sensors for human detection and localization, there are also important challenges that need to be addressed [30]. The most critical one is related to the false alarms due to noise or multipath components (MPC). Indeed, since the MPC magnitude is significant, the MPC may often be confused with backscattered signals from humans.

To differentiate the multipath signal from real target signal, three categories of approaches have been proposed in the literature, as follows:

1) Approaches relying on features extracted from the time of arrival (ToA) information of LoS and NLoS signal components, such as the backscattered signal mean and variance [31], [32]. The problem with this type of classification is setting an appropriate threshold, which might vary in different environments.

2) Approaches based on the signal propagation path loss model. The main assumption considered by these approaches is that the energy level of the first (direct) path is greater than that of the MPC. Kurtosis, peakto-lead delay, mean excess delay and root mean square (RMS) delay spread, as well as some other features are extracted and fed to various classifiers, such as support vector machine (SVM), multi-layer perceptron (MLP), 
and decision tree [5], [33]-[35]. However, due to some factors such as a heavy cluttered indoor environment, the distance between the transmitter and the receiver or the antenna height, the propagation path loss model may not be well suited and hence, the manually selected features could not be sufficient to obtain robust classification results.

3) Approaches using deep learning based methods to overcome the classification difficulty due to manually selected features. For instance, in [36], a CNN has been used to distinguish LoS/NLoS signal components. The main problem associated with deep learning based classification is that it requires a huge amount of training and evaluation data, which is time consuming and laborintensive. Moreover, the accuracy of deep learning approaches in MPC removal [36] may not be enough for safety critical purposes.

Consequently, relying on the aspects introduced above, we focus in this paper on removing the MPC by data fusion from distributed multiple radars. In related work [37], [38], the authors have used multiple-input multiple-output (MIMO) radar set-up and a maximum likelihood method for MPC removal, but the paper is based on simulations and parameters optimization rather than on realistic data. Our main goal is to detect people and moving machines in heavy cluttered environments, with noise and MPC, as well as to localize and classify them in order to avoid any collision between human and machines in a hybrid industrial environment. When the radar transmitted signal is backscattered from the environment, it contains unwanted clutter components. Signal pre-processing steps such as clutter removal, signal envelope detection and compensation of the distance effect are applied to the raw reflected signal. After signal pre-processing, a multipulse cell averaging constant false alarm rate (MCA-CFAR) algorithm is applied to detect humans or moving machines in a way to reduce the misdetections resulting from noise or heavy cluttered environment. In order to reduce the MPC, an algorithm is proposed to compare the positioning error of multiple peak components from multiple radar set-ups. A joint probabilistic data association (JPDA) tracker is used to initialize and delete tracks and associate data received from multiple radar sensors to each track [39]. In this work, a cart is chosen as a generic object to represent a mobile machine in an industrial indoor environment. Such a machine can also have rotating parts such as stepper motors or servo motors to control its motion [40], [41]. We have emulated these rotating parts with a small fan placed on the cart. In the collision avoidance problem, the targets may be stationary or moving, which makes them difficult to classify with conventional features based classifiers. Some machine learning based research results have been previously reported [42], but with experiments conducted in a very controlled environment where the subjects were stationary. In a related work [43], a deep learning based classification method was used for differentiating a human from a vehicle on road. In this paper, a CNN classifier is used to extract salient features and discriminate the cart from human targets. The main contributions of our work can be summarized as follows:
1) An MCA-CFAR algorithm is introduced for UWB radarbased people detection to reduce the noise and clutter components. A human free background allows extracting the reference clutter signal for the MCA-CFAR algorithm, which provides good performance in terms of reducing scattered noise peaks as it will be shown in Section III. The result of MCA-CFAR contains signal components reflected from human/moving machines as well as MPC.

2) An enhanced measurement set-up, using four radar sensors instead of three in a standard configuration, is defined to enhance the positioning results by reducing the MPC. The additional radar sensor is used to compare object positions yielded by multiple radar set-ups (each set-up consists of three radars) and only the peaks leading to consistent positions are retained.

3) A cart with a fan is used to emulate a generic moving machine in a hybrid industrial environment. To differentiate the moving machine from humans, a CNN classifier is employed. Positioning results are included to show the accurate positioning of multiple targets in indoor environments. The performance of the proposed algorithms is assessed in real time using practical data measured with radar sensors. Various parameters used in the proposed algorithms are optimized using measured radar data.

The remainder of the paper is organized as follows. Section II deals with the signal pre-processing steps and system overview of the proposed scheme for multi-human detection, localization and classification of moving machines and human targets. In Section III, the MCA-CFAR algorithm is detailed and validated using real data from UWB radar sensors. Next, sensor fusion of multiple radars set-up is discussed to reduce the multipath effect in Section IV. In Section V, the classification of a generic moving machine from humans is discussed. The experimental results are analyzed in Section VI. Conclusions are drawn in Section VII, which also includes some ideas for future work.

\section{SYSTEM OVERVIEW AND SIGNAL PRE-PROCESSING}

In this paper, a set-up based on mono-static radar array is considered for multi human/moving machine detection, localization and classification. Four UWB radar sensors, with known positions, are placed on two orthogonal axes, as shown in Fig. 1. This specific geometry choice is made because of simplicity of the sensors deployment and coverage of the whole area within the room. The signal processing steps are illustrated by the system overview given in Fig. 2 .

The received signal is first filtered with clutter removal filter. Then the thresholding is performed for one time step using a novel MCA-CFAR algorithm. After thresholding, the MPC are removed by using an algorithm based on four radars setup. Then localization and classification tasks are performed. The signal pre-processing step is described below and the remaining steps are explained in the following sections. The received signal is first pre-processed, so that unwanted clutter 


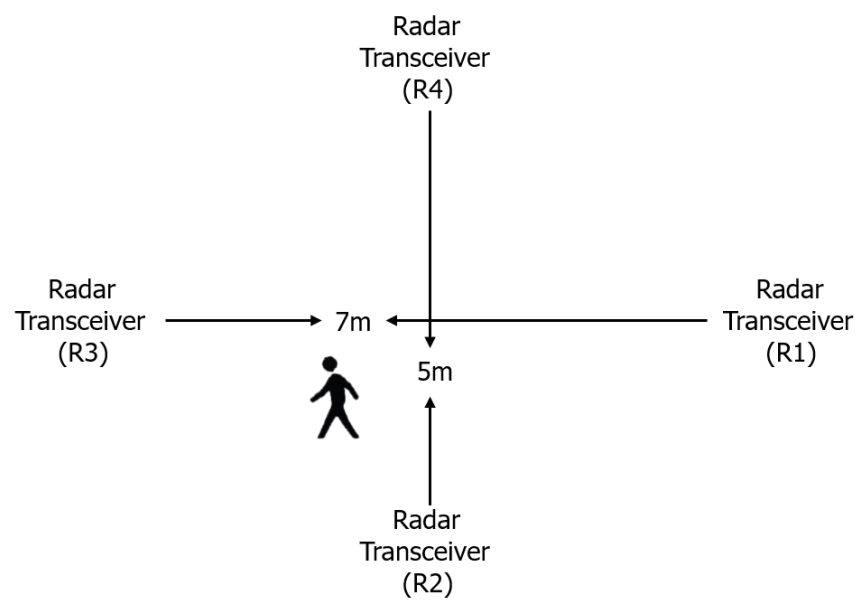

Fig. 1: Mono-static radar array set-up for humans/moving machines localization and detection.

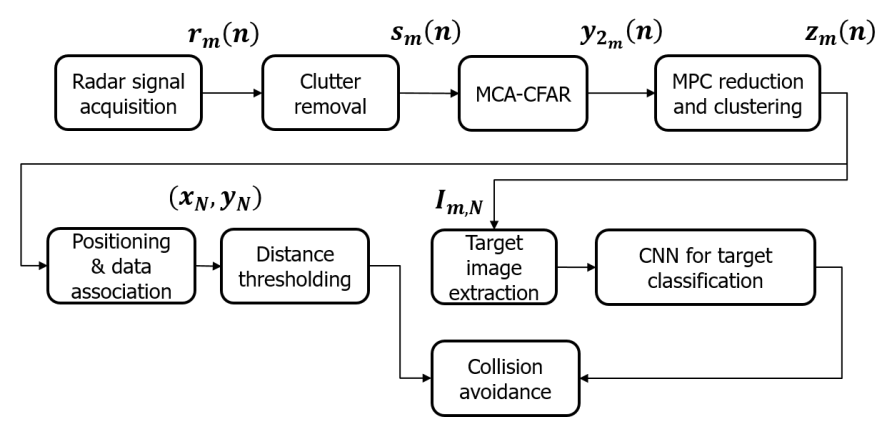

Fig. 2: System overview.

and noise can be removed from the signal. The reflected signal is represented as:

$$
r_{m}(n)=\sum_{l=1}^{L} a_{m l} p\left(n-\tau_{m l}\right)+\mathcal{N}(n)
$$

where $n$ and $m$ stand for the fast time and slow time index respectively, while $\tau_{m l}$ and $a_{m l}$ are the time delay and the coefficient associated with the $l^{\text {th }}$ multipath. $p(n)$ and $\mathcal{N}(n)$ represent the elementary radar waveform and additive noise, respectively. The fast time refers to the received signal variation during each transmission period and is directly related to the slant range from the radar, while the slow time is associated to the signal variation over several successive transmission periods. The backscattered raw signal $r_{m}(n)$ contains the reflection from all the objects in the environment. In order to remove the clutter components from the backscattered signal, an averaging filter is used [44]. The simple averaging filter may be represented as follows:

$$
\begin{gathered}
c_{m}(n)=\alpha c_{m-1}(n)+(1-\alpha) r_{m}(n) \\
s_{m}(n)=r_{m}(n)-c_{m}(n)
\end{gathered}
$$

where $\alpha$ is proportional to the signal to clutter ratio, $c_{m}(n)$ represents the clutter signal, and $s_{m}(n)$ is the filtered signal after removal of the clutter components. A lower value of $\alpha$ results in a faster estimation of the environment clutter,

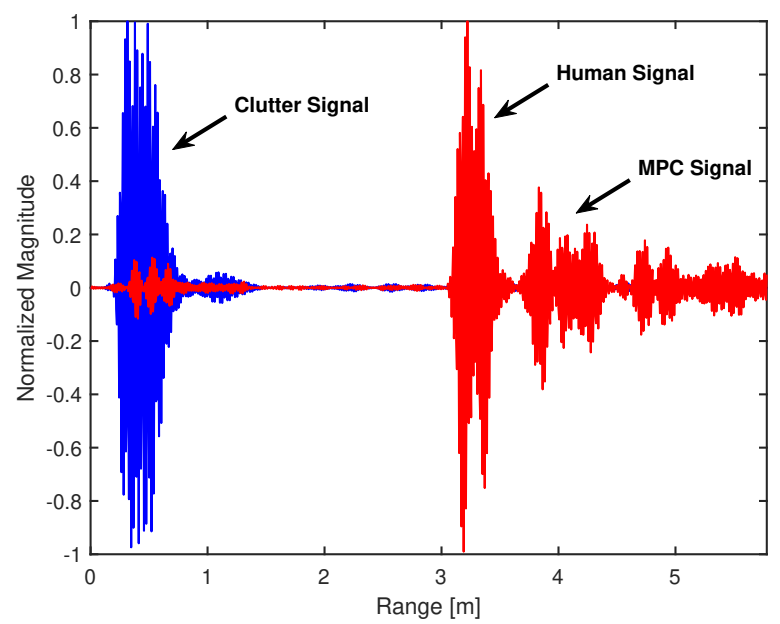

Fig. 3: Normalized raw and filtered signals after clutter removal.

but the clutter signal may be affected by impulse noise. Comparatively, a higher value of $\alpha$ needs longer time for clutter estimation, but it makes clutter estimation robust to impulse noise [45]. The radar raw and filtered signals for the case of a standing person are represented in Fig. 3.

In Fig. 3, it may be noticed that the raw signal, represented by blue line, has strong clutter component at around $0.5 \mathrm{~m}$ distance due to reflections inside the room, and hence, the useful signal from the standing human is not observable. After clutter removal, the signal from human is clearly observable at around $3.25 \mathrm{~m}$ distance, as shown by the red line in Fig. 3 . It can also be noticed that there are multipath components located on the right side of the human peak. The filtered signal is then passed through envelope detection and distance compensation blocks. After the pre-processing stage, a MCACFAR algorithm is employed for human detection.

\section{MCA-CFAR ALGORITHM}

In this paper we consider a MCA-CFAR algorithm for human detection. The standard CA-CFAR algorithm [46] makes the decision for the current range cell using a detection threshold calculated from the clutter signal, measured in the neighboring range cells. However, this scheme is not suitable when the neighboring range cells also contain backscattered signals from other people in a dense environment such as smart factory. Therefore, we have collected data from the human free room, and used the envelope of the corresponding reference signal to calculate the detection threshold for each range cell. The probability density function (PDF) of the reference signal envelope has been then estimated, as shown in Fig. 4, from the data collected in the range cells at multiple pulses. By comparing the estimated PDF to several competing distributions, we found out that the Gamma PDF is the best fit to the collected data. This hypothesis has also been validated using the Kolmogorov-Smirnov test, with a significance level of 5\%. Based on the expressions of the Gamma distribution moments [47], the shape and rate parameters of the clutter PDF 


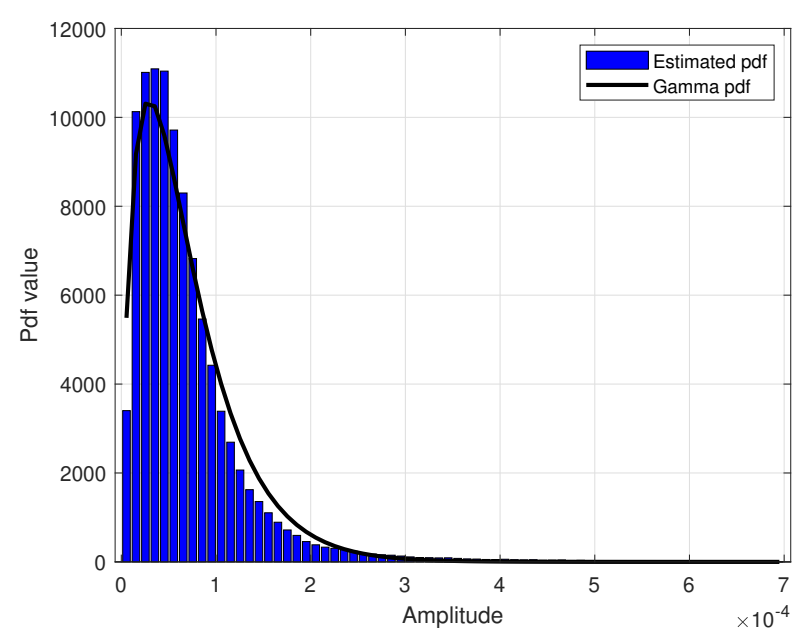

Fig. 4: Estimated PDF of the reflected signal (blue bars) and a Gamma distribution fit for the data (black line).

can be calculated using the estimated mean $\hat{m}_{s}$ and variance $\hat{\sigma}_{s}^{2}$ of the reference signal as:

$$
\left\{\begin{array}{l}
\hat{\alpha}=\frac{\hat{m}_{s}^{2}}{\hat{\sigma}_{\hat{\hat{y}}}^{2}} \\
\hat{\theta}=\frac{\hat{\sigma}_{s}}{\hat{m}_{s}}=\frac{\hat{\alpha}}{\hat{\alpha}}
\end{array}\right.
$$

A second " $K$ over $N$ " detection stage is then introduced to remove the spurious, clutter related peaks, at the output of the first detection stage. Let us denote the binary decision at the detector output by $\delta_{0}$ (clutter only) and $\delta_{1}$ (clutter + human). The MCA-CFAR algorithm consists in accumulating in slow time $N$ successive binary decisions in a given range cell, provided by the first detection stage, and deciding $\delta_{1}$ if there are at least $K$ positive detection results among them.

The key result, which allows controlling the overall false alarm probability, $P_{\mathrm{fa}}$, is that the binary decision at the output of the first detection stage follows a binomial distribution. It is thus possible to link the false alarm probability at the output of the first detection stage $P_{\mathrm{fa} 0}$ to the overall false alarm probability as shown in (5), where $\left(\begin{array}{l}N \\ k\end{array}\right)=\frac{N !}{k !(N-k) !}$ is the binomial coefficient:

$$
P_{\mathrm{fa}}=\sum_{k=K}^{N}\left(\begin{array}{c}
N \\
k
\end{array}\right) P_{\mathrm{fa} 0}^{k}\left[1-P_{\mathrm{fa} 0}\right]^{N-k}
$$

A similar relationship is established in (6) between the detection probabilities $P_{\mathrm{d} 0}$ and $P_{\mathrm{d}}$ at the output of the first and second detection stage, respectively.

$$
P_{\mathrm{d}}=\sum_{k=K}^{N}\left(\begin{array}{c}
N \\
k
\end{array}\right) P_{\mathrm{d} 0}^{k}\left[1-P_{\mathrm{d} 0}\right]^{N-k}
$$

This step ensures that if a peak is due to a human or other moving object, then it must stay for certain pulses in slow time due to the reasonable speed of the object movement. $N$ and $K$ are adjustable and can be adapted to the normal speed of the human or other targeted objects for detection.

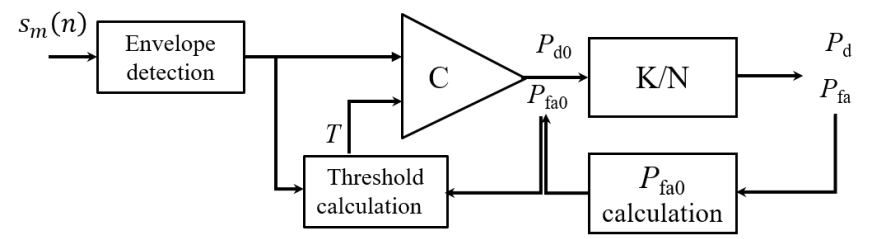

Fig. 5: Block diagram for the two-stage multi-pulse MCACFAR algorithm.
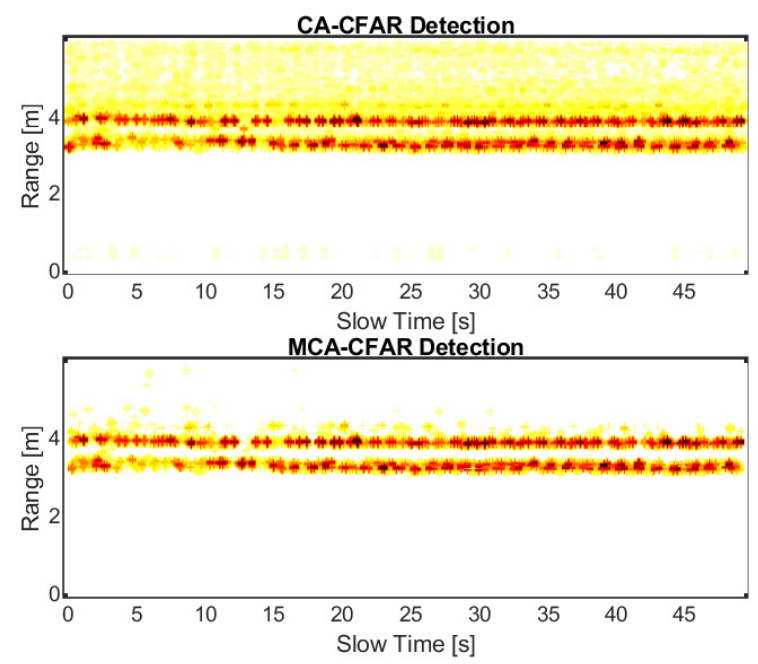

Fig. 6: CA-CFAR and MCA-CFAR based detection result of a simple scenario.

The block diagram in Fig. 5 represents the functionality of the MCA-CFAR. The comparator " $\mathbf{C}$ " and the block "K/N" make the decisions in the two stages of the detection scheme. In each range cell the second stage makes a decision based on the number of positive detection at the output of the first stage, within the $N$-length window along the slow time. The detection threshold $T$ can be determined to obtain the false alarm probability $P_{\text {fa0 } 0}$ at the output of the first stage, using (7):

$$
T=G_{\hat{\alpha}, \hat{\theta}}^{-1}\left(P_{\mathrm{fa} 0}\right)
$$

where $G$ stands for the Gamma cumulative distribution function (CDF).

A pseudo-code description of the MCA-CFAR processing flow is given by Algorithm 1.

To evaluate the performance of the proposed algorithm, two experiments were conducted. The first experiment consisted of a simple scenario when a person was standing in the room. The true position of the person is at around $3.4 \mathrm{~m}$ from the radar. A multipath component at around $4 \mathrm{~m}$ is generated and can be observed from the Fig. 6. The results of the CA-CFAR algorithm and the MCA-CFAR algorithm are shown in this figure.

As illustrated in Fig. 6, it is clear that the proposed MCACFAR result has very few noisy peaks compared to the CACFAR detection result. Note that although both the noise and clutter are reduced by employing the MCA-CFAR algorithm, 
Algorithm 1 Proposed MCA-CFAR algorithm

Input:

$N$ : number of successive pulses over which the final decision is made.

$K$ : number of positive detection results over the $N$ successive pulses, at the output of the first detection stage, required to decide $\delta_{1}$.

$P_{\mathrm{fa}}$ : required false alarm probability at the output of the MCACFAR algorithm.

$s_{k}(n)$ : backscattered signal from the $k^{\text {th }}$ transmitted pulse, where $k=1, \ldots, N$, and $n$ denotes the current range cell in fast time.

\section{Output:}

Detection decision: $\delta_{0}$ or $\delta_{1}$.

\section{Data processing:}

1) Perform the envelop detection of the signal $s_{k}(n)$ and denote the output signal by $S_{k}(n)$.

2) Estimate the shape and rate parameters $\{\hat{\alpha}, \hat{\theta}\}$ of the Gamma distributed clutter using (4).

3) Determine $P_{\text {fa } 0}$ by solving (5) for the given values of $N, K$ and $P_{\text {fa }}$.

4) Calculate the detection threshold $T$ using (7).

5) Initialize $\Sigma_{n}=0$ and go through the following loop:

For $k=1: N$

If $\left(S_{k}(n) \geq T\right)$

\section{End if}

Increment $\Sigma_{n} \leftarrow \Sigma_{n}+1$,

\section{End for}

6) Make the final decision:

If $\left(\Sigma_{n}<K\right)$

Decide $\delta_{0}$.

Else

End if

\section{Decide $\delta_{1}$.}
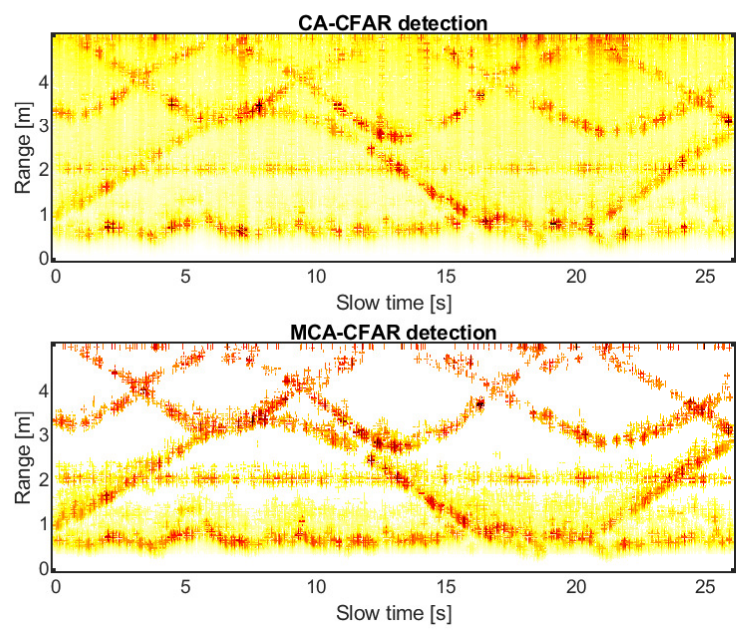

Fig. 7: CA-CFAR and MCA-CFAR based detection result of a complex scenario.

the strong MPC still exist and need to be removed for accurate multi-human localization. The MPC has a strong value and it has a strong density as well, therefore it is very difficult to differentiate it from the actual target by using thresholding techniques such as CA-CFAR or MCA-CFAR. This challenge will be tackled in the section IV. The next experiment was conducted in relatively large hall with five targets. One machine and four humans were involved in that experiment. Three persons were moving, whereas one person and the machine were located at a fixed distance from the radar. From Fig. 7, it is clear that the proposed MCA-CFAR algorithm results in a fewer noisy peaks as compared to the conventional CA-CFAR algorithm.

\section{SENSOR DATA FUSION FOR MINIMIZING THE MULTIPATH EFFECT}

In this section, we introduce a strategy based on an array of mono-static radar sensors to reduce the multipath effect. A previous work [38] has shown by simulating various numbers of transmitter $(\mathrm{Tx})$ receiver $(\mathrm{Rx})$ pairs, that more Tx-Rx pairs result in reduced overall positioning error. In this work, we have used four radar sensors and two radar set-ups. Each setup comprises of three radar sensors. The three radars for each set-up are chosen randomly. The first combination of three radars is used to find the candidate targets. For a 2D scenario, every time of arrival (ToA) represents a circle. The circles produced by the ToAs corresponding to the direct path (DP) to the target intersect at a single point (or small intersecting area), i.e., the location of actual target, yielding a small estimation error. Contrariwise, the circles that correspond to the ToAs from different targets, MPC or clutter peaks result in larger intersection area. An example is shown in Fig. 8 to show the above observation.

The experimental scenario of Fig. 8 consists of three radar sensors used for measurement of distance of the target. A target is present in the range of the three radars and the backscattered signals contain the target as well as the multipath signals. In Fig. 8a, the locations of peaks for the three radars $\mathrm{R} 1, \mathrm{R} 2$ and $\mathrm{R} 3$ are $3.2,2.1$ and $3.1 \mathrm{~m}$, respectively. It may be noticed that the clustered peak locations which refer to radar ranges from the target object almost overlap with the distances estimated from the Least Squares (LS) algorithm [48]. The estimation error value obtained by (10) in this case is $5.4 \mathrm{~cm}$. It can be concluded that all the ranges involved in this combination are from the human and that they do not include any multipath component. In Fig. 8b, the locations of peaks for the three radars R1, R2 and R3 are 4.0, 2.1 and $3.3 \mathrm{~m}$ respectively. This combination involves multipath components, which result in a much larger estimation error $(79.8 \mathrm{~cm})$. It may also be noticed in Fig. $8 \mathrm{~b}$ that the radar ranges obtained after clustering and the distances estimated by LS algorithm are not overlapping, as they correspond to only human reflected signals. In the above example, one radar setup i.e. R1-R2-R3 was used for MPC removal. However, since there may be some ToAs combination from different targets, MPC or clutter which also result in small intersecting area, we use another set-up of three radars to refine the results. A candidate target is considered to be a valid estimate if the ToAs from both radar set-ups result in small intersecting areas 


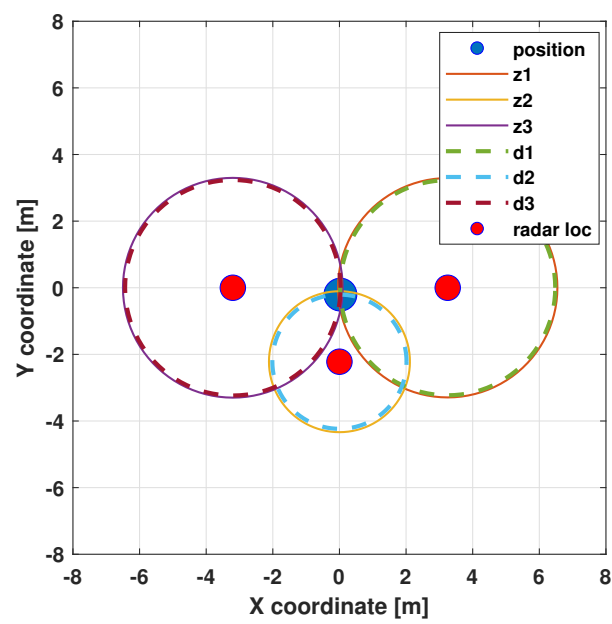

(a)

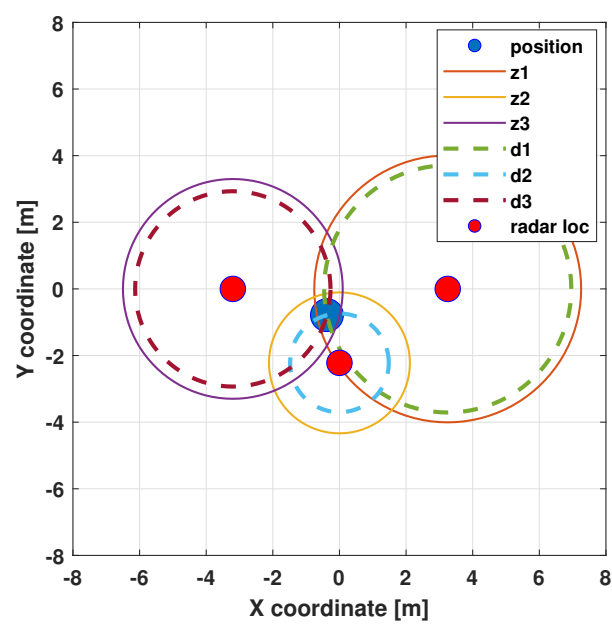

(b)

Fig. 8: Trilateration and distance estimation for the case of (a) only LoS signal components (b) when there are MPC involved.

(or less estimation error). The proposed setting based on four radar sensors is shown in Fig. 9.

The setting in Fig. 9 consists of two overlapping radar set-ups: set-up A (R1-R2-R3) and set-up B (R1-R3-R4). The peak locations are chosen as human or MPC based on the positioning error from different radar set-ups. A block diagram of the proposed algorithm is shown in Fig. 10.

In Fig. $10, z_{i}(n), i=1, \ldots, 4$ represent the clustered peak locations after applying the MCA-CFAR algorithm. The details of clustering algorithm can be found in the paper [45]. $e_{\text {est }}$ and $e_{\text {est }}$ stand for the position estimation errors, which may be calculated by (10) and $\gamma$ represents the error threshold. The threshold value $\gamma$ is selected experimentally. Actually, there is a tradeoff to be found as high values of $\gamma$ result in increased overcounting and vice versa. A detailed step by step explanation of the processing flow is given by Algorithm 2 .

Algorithm 2 summarizes the MPC reduction at only one slow time of the radar signal. Therefore, this procedure will be repeated for the given number of slow time indices.
Algorithm 2 Proposed multiple radar set-ups based algorithm for MPC reduction.

Input: $y_{2_{m}}^{i}(n), i \in 1,2,3,4$

Output: $\left(x_{u}, y_{u}\right): 2 \mathrm{D}$ coordinates of the $N$ detected targets Clustering:

The peak locations which are close to each other are clustered as they originate from the same object, like in [22]. $z_{i}(n)$ represents the output of the clustering algorithm.

\section{Initialize:}

$u \leftarrow 1 ; k \leftarrow 1$

$p_{t}$ : Total number of clustered peaks locations.

While $\left(k \leq p_{t}\right)$

\section{Positioning error calculation:}

a) Position estimation $\left(\tilde{x}_{k_{1}}, \tilde{y}_{k_{1}}\right)$ by set-up A (R1-R2-R3):

$$
z_{i, k}^{2}=\left(x_{k_{1}}-x_{i}\right)^{2}+\left(y_{k_{1}}-y_{i}\right)^{2}, i \in\{1,2,3\}
$$

In (8), $x_{i}$ and $y_{i}$ represent the radar coordinates. The circles made by the distances from the radars may not intersect at one point but in a region. To select the optimum point inside the intersecting region, Least Squares (LS) estimation [48] is used. By linearizing the above set of equations and using LS, the approximate values of the $2 \mathrm{D}$ coordinates $\tilde{x}_{k_{1}}$ and $\tilde{y}_{k_{1}}$ of the candidate target are determined. Since the approximated position lies inside the intersection region, therefore the distance of this position from each radar may be different than the original distances of target from the radar.

b) Distance calculation of the approximated position from each radar:

The distance of the approximated position $\left(\tilde{x}_{k_{1}}, \tilde{y}_{k_{1}}\right)$ to each radar i.e. $d_{\text {est }_{i, k}}$ is calculated by (8) as follows.

$$
d_{\mathrm{est}_{i, k}}=\sqrt{\left(\tilde{x}_{k_{1}}-x_{i}\right)^{2}+\left(\tilde{y}_{k_{1}}-y_{i}\right)^{2}}, i \in\{1,2,3\}
$$

c) Positioning error calculation:

If the circles corresponding to radar ranges, i.e., the clustered peak locations $z_{i, k}$ intersect in a small region, then the difference between recalculated distances $d_{\text {est } t_{i, k}}$ and the original radar ranges $z_{i, k}$ will be small and vice versa. Positioning error is calculated by (10).

$$
e_{\mathrm{est}_{1}}=\sqrt{\left(z_{1, k}-d_{\mathrm{est}_{1, k}}\right)^{2}+\left(z_{2, k}-d_{\mathrm{est}_{2, k}}\right)^{2}+\left(z_{3, k}-d_{\mathrm{est}_{3, k}}\right)^{2}},
$$

Find the positioning estimation error $e_{\text {est }}$ for the radar set-up B, using (8), (9) and (10).

If $\left(e_{\text {est }_{1}}<\gamma \& e_{\mathrm{est}_{2}}<\gamma\right)$

Save $z_{i, k}$.

Perform localization by trilateration to find $\left(x_{u}, y_{u}\right)$

End

$u \leftarrow u+1$

$k \leftarrow k+1$;

End while 


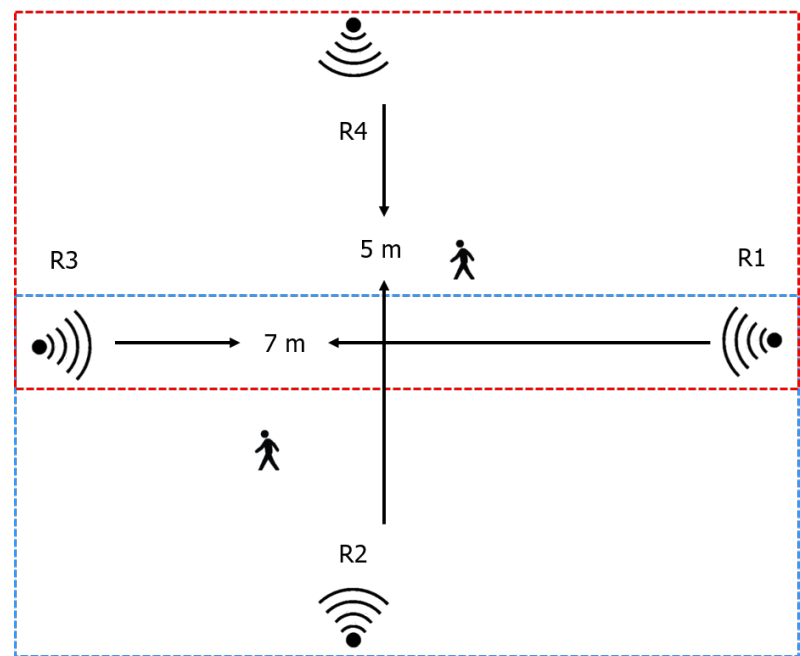

Fig. 9: Four radar set-up to make 2 planes in $2 \mathrm{D}$ for reducing MPC.

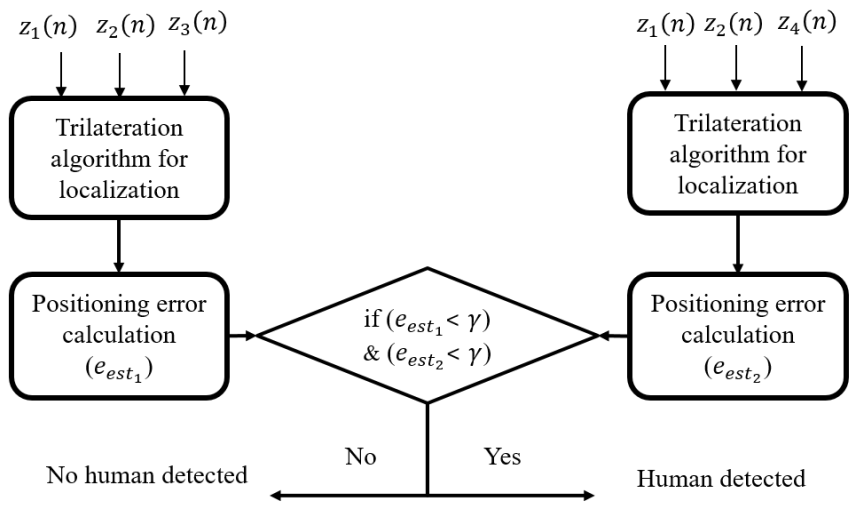

Fig. 10: Algorithm for removal of MPC.

\section{TARGET CLASSIFICATION ALGORITHM}

In a collision avoidance problem, the human and machine do not have fixed radar cross section (RCS), velocity and shape. Different humans have different physical structures. Similarly, the RCS and shape of the industrial moving machines may change. For instance, for the cart considered in this paper as a generic moving machine, its RCS and shape depend on the objects carried with it. Therefore, the classification algorithms that extract the above mentioned features or features derived from these main features may not work robustly. To provide a robust classification output, we use an image-based classifier. These classifiers extract the pattern from the image automatically. In this work, a deep CNN, which does not rely on hand crafted features, is used for classification. The cart with a rotating fan shown in Fig. 11 is used in our experiments to emulate a generic moving machine in an industrial indoor environment.

After applying the thresholding and multipath removal algorithms, the matrix of clean signals is then converted into an image as follows. In order to prepare the input images for the classification algorithm, first we extract the region of interest (ROI) from the clean radar frames. Since each target is represented by a cluster as shown in the MPC removal algorithm, we have to extract the portion of the signal which is associated with the target and ignore the rest of the signal. We have chosen the ROI that corresponds to the target radar cross section, as $50 \mathrm{~cm}$ for the image length. The value of image length is chosen based on the target size. If the machine size is huge then this value may even be increased for optimal classification results. The image width that corresponds to the slow time, is chosen as 2 seconds or 100 frames. By decreasing it, the accuracy was lower and if it is further increased then the time delay is larger without much accuracy improvement. Another factor that decides the number of frames for the image width is the slow time sampling frequency of the acquisition system. The center of the target cluster is located at each time step and the ROI is extracted by selecting the specific number of bins below and above the center of cluster as shown in Fig. 12a. In $12 b$, an example of ROI for an experiment is shown. In that experiment a machine is located in front of the radar at a distance of approximately $50 \mathrm{~cm}$. Next, the ROI is transformed into a grayscale image as shown in $12 \mathrm{c}$. In the grayscale image the intensity of the pixels varies from 0 (white) to 255(black). These images are then stored in respective folder. One folder stores the images of human target while the other folder stores the machine images.

The layers used in our CNN are described in the Fig. 13. The convolutional layers, designed to extract salient features, are followed by pooling layers, which reduces their output size through subsampling techniques. Our CNN includes three convolutional layers and two pooling layers. The hyper parameters, such as filter sizes of convolutional layer, are chosen based on trial and error method until we obtained a desirable accuracy. The rectified linear unit (ReLU) function is selected for activation because of its better performance, in the considered framework, compared to tanh and sigmoid functions. Similarly, we have chosen max pooling in the pooling layer due to its superior performance for our problem, compared to other pooling methods, such as mean or median pooling. A fully connected layer is also considered for the classification based on the output of the last convolutional layer. As there are two output classes in our case, the output will be either a human or a cart target. 


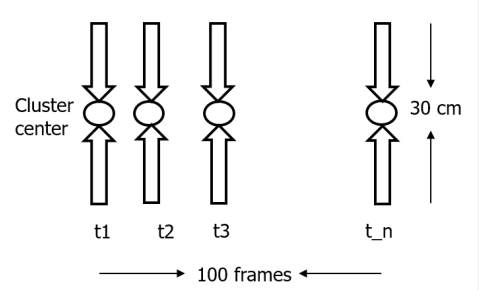

(a)

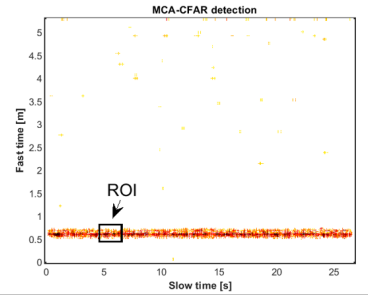

(b)

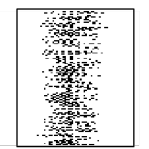

(c)

Fig. 12: Method of (a) ROI extraction (b) Example of ROI (c) Grayscale image conversion.

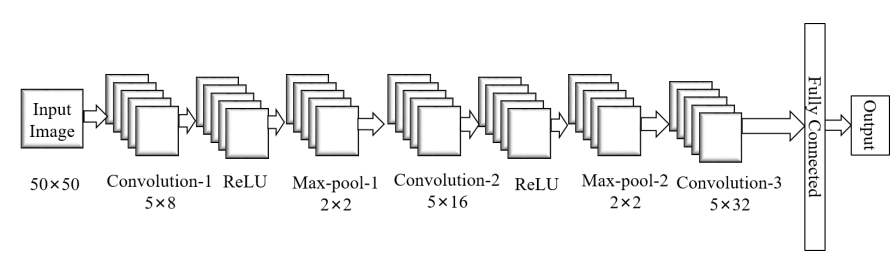

Fig. 13: Deep CNN structure for targets classification.

\section{RESULTS AND DISCUSSION}

\section{A. Experimental design}

In order to assess the performance of the proposed techniques, we have performed real time experiments with IRUWB transceivers. The hardware set-up of the system consists of four radar sensors (Novelda Xethru X4), placed on the walls of the room at fixed locations and connected to a PC. The MatLab software was used for processing the reflected signals from the radar sensors. The transceiver timing is derived from a crystal oscillator running at $27 \mathrm{MHz}$, and a 243-MHz PLL generates a system clock that is used for state machines, and as a common clock for the TX and RX PLLs. It is also used for synchronizing multiple radar sensors [49]. The radar sensor specifications are given in Table I [50].

The experiments in this work were performed on different days and with different clutter environments to show that the proposed algorithm is robust against any clutter changes in the environment. Three sets of experiments were performed. The first one was performed in an indoor environment, i.e., a cluttered room (SET-UP A in the following), while the second and third ones were carried out in a lab environment (SET-UP $\mathrm{B}$ and $\mathrm{C}$ in the following).

SET-UP A:

A room was considered because the MPC are much stronger than for a large open hall or outdoor environment, due to the relatively small distance between the walls. Fig. 14 illustrates the set-up A and shows only two out of the four radar sensors deployed along the four walls. The distances between the radar sensors are given in Fig. 9. In these experiments,

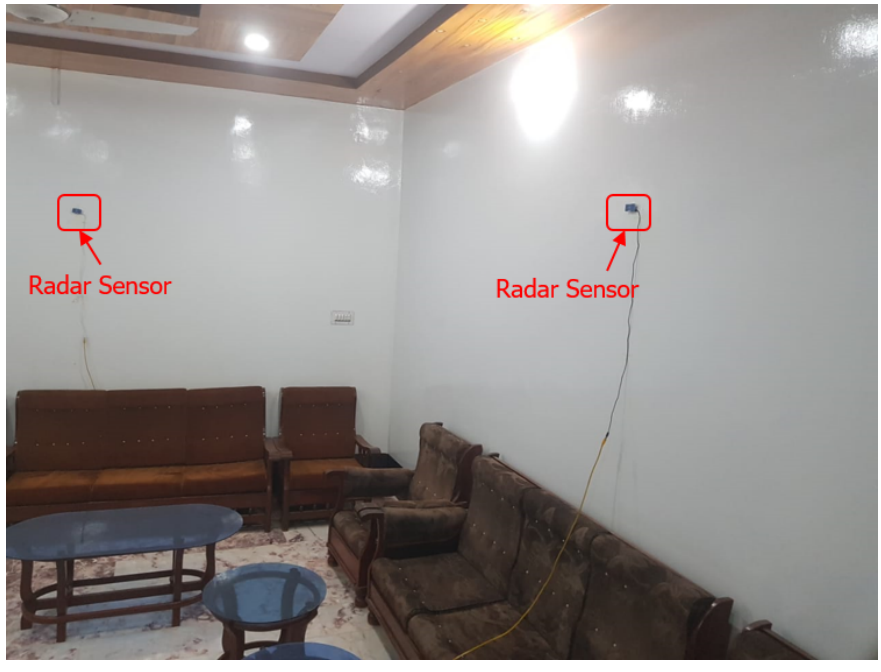

Fig. 14: Experimental set-up A.

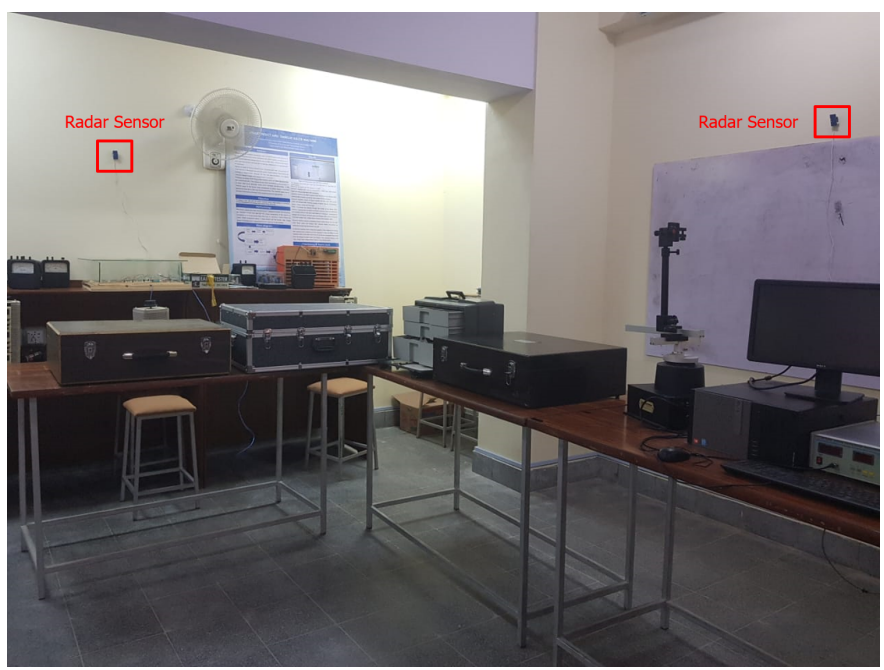

Fig. 15: Experimental set-up B and C.

scenarios involving only one or two persons were considered to illustrate the performance of the proposed MCA-CFAR and MPC reduction algorithms.

SET-UP B and C:

The next set of experiments was performed inside a lab with furniture and metallic equipment to assess the performance of the proposed algorithms with a large number of participants, as compared to the first set of experiments. These experiments involved both human subjects as well as a moving cart. The set-up B is depicted in Fig. 15, which shows again only two out of the four radars placed on the four walls of the lab. The coordinates of the radar sensors may be also found in Fig. 9. Lastly, another set of experiments were carried out in the same lab environment to evaluate the results of the deep CNN for distinguishing humans from the moving machines. In the second and third set of experiments there were five targets, i.e., four people and a cart.

The details of the targets involved in our experiments are shown in Table II. Some of the parameters were chosen experimentally such as gain of clutter removal filter, error 
TABLE I: Radar sensor parameters.

\begin{tabular}{|c||c|}
\hline \multicolumn{1}{|c||}{ Parameter } & Value \\
\hline Output power & $-12.6 \mathrm{dBm}$ \\
\hline Center frequency & $8.7 \mathrm{GHz}$ \\
\hline Pulse repetition frequency & $400 \mathrm{MHz}$ \\
\hline Sampling frequency & $23.3 \mathrm{GHz}$ \\
\hline Slow time sampling frequency & $30 \mathrm{~Hz}$ \\
\hline
\end{tabular}

TABLE II: Details of the targets involved in the experiments.

\begin{tabular}{|c||c||c||c||c|}
\hline $\begin{array}{c}\text { Subject } \\
\text { type }\end{array}$ & $\begin{array}{c}\text { Gender } \\
\text { (M/F) }\end{array}$ & Age (years) & Weight (kg) & Height (cm) \\
\hline Human & M & 33 & 75 & 182 \\
\hline Human & F & 26 & 63 & 170 \\
\hline Human & M & 21 & 71 & 173 \\
\hline Human & M & 28 & 78 & 174 \\
\hline Cart & N/A & N/A & 30 (with load) & 105 \\
\hline
\end{tabular}

TABLE III: Parameter values chosen for the experimental setup.

\begin{tabular}{|c||c|}
\hline Parameter & Value \\
\hline$\alpha$ (clutter removal) & 0.7 \\
\hline Exponent in distance compensation & 1.7 \\
\hline$N, K$ & 9,7 \\
\hline$\gamma$ & $15 \mathrm{~cm}$ \\
\hline
\end{tabular}

threshold and distance compensation exponent. The parameter values chosen for the experimental set-up are given in Table III.

\section{B. Detection results (SET-UP A)}

The first set of experiments are performed in experimental set-up A and the following scenarios are considered.

- Scenario 1. One person standing: In this scenario, only one person is standing in the room The position of the person is almost same throughout the slow time with only minor routine movement of a human.

- Scenario 2. One person walking: In this scenario, a single person is walking in the room. The speed corresponds to normal walking at around $0.5 \mathrm{~m} / \mathrm{s}$. The motion is made along a straight line in the center of the rectangular area.

- Scenario 3. Two persons standing: In this scenario, two people are standing in the room. The individuals were standing at fixed locations while performing some natural motions such as moving the head a little or tilting the body left and right.

- Scenario 4. Two persons walking: In this scenario, two people are moving in different directions. The motion speed was similar to the scenario 2 .

1) MCA-CFAR detection results: The backscattered radar signals are passed through pre-processing stages to remove clutter signal and compensate for the effect of signal magnitude degradation due to increasing distance from the radar. Next, the MCA-CFAR algorithm is applied to the preprocessed signals. The MCA-CFAR results of radar R2 for the four scenarios are shown in Fig. 16. It is worth noting that results for the other three radars are similar.

As shown in Fig. 16, in case of Scenario 1, the MCA-CFAR detection result show the human peak cluster at around $2 \mathrm{~m}$, while at around $3 \mathrm{~m}$ there is strong MPC which is not desirable
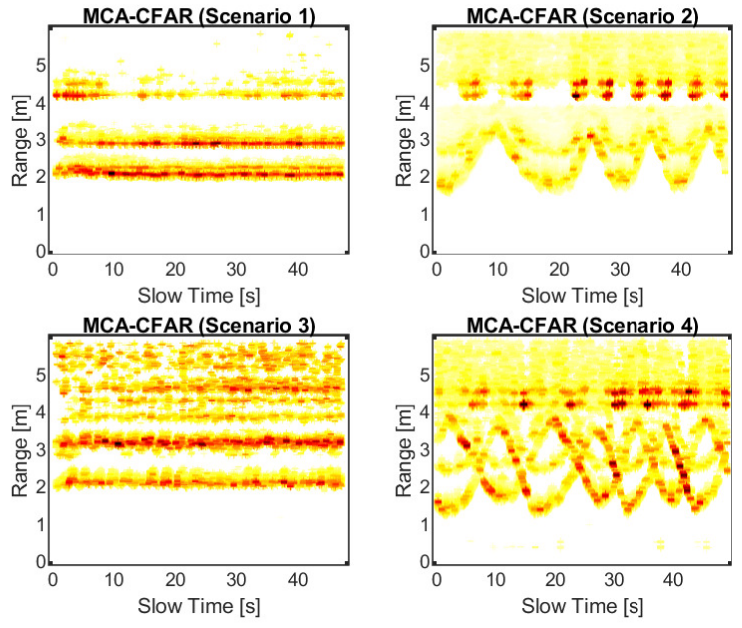

Fig. 16: MCA-CFAR results of R2 for four scenarios.
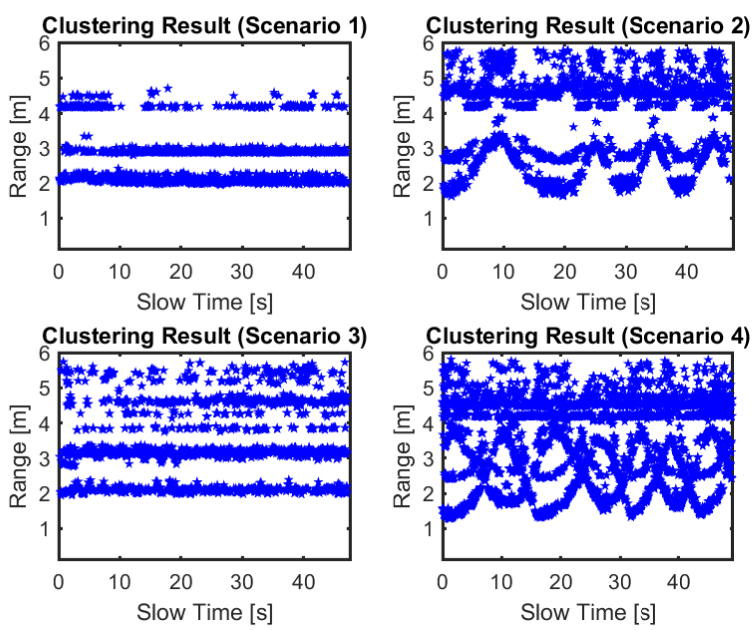

Fig. 17: Clustering result of radar R2 for the four scenarios.

for reliable human detection. As the radar signal suffers from path loss, in order to compensate it, the samples that are further from the radar are weighted by high values [51], which make it stronger. In some cases, the MPC become even stronger than the human peaks due to the path loss compensation, because MPC are located further than the human peaks. In addition to MPC, there are also strong clutter and noise components present in the signals such as in Scenario 1 at around $4 \mathrm{~m}$ distance. These strong clutter components are mainly due to the walls and floor of the room. The peak values are then clustered as shown in the following section.

2) Clustering results: The MCA-CFAR results obtained above contains clusters of peak values. The moving objects have larger radar cross-section (RCS) and one object reflects the signal which may be spread along the fast time axis. Thus the locations of the peaks need to be clustered together, as shown in Fig. 17. The clustered peaks in this figure have MPC, such as the signal component at $3 \mathrm{~m}$ for Scenario 1. These clustered peaks also have clutter residuals such as the signal components in the range 4-6 m for Scenario 2. In order to 

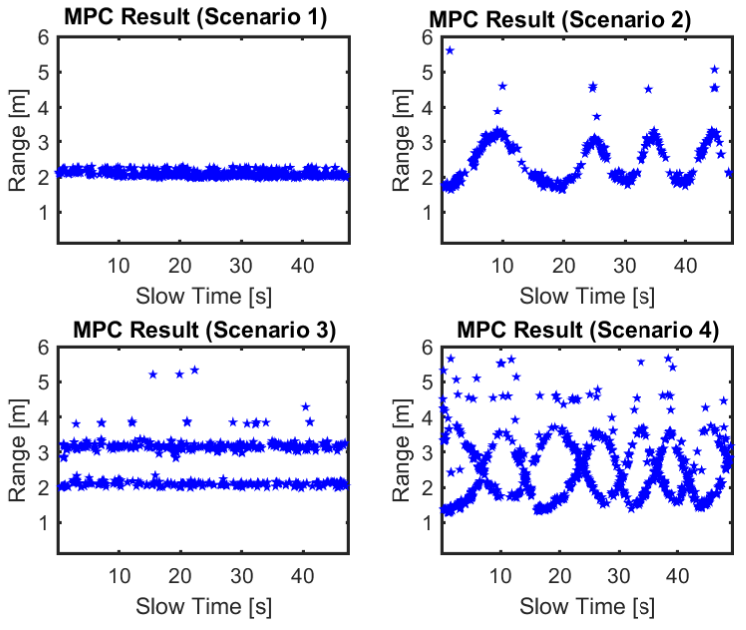

Fig. 18: MPC removal algorithm results of radar R2 for four scenarios.

reduce the MPC as well as the clutter residuals, these clustered signals are then passed through the proposed MPC removal algorithm based on multiple radar set-ups as shown in the next section.

3) Results after MPC removal: From Fig. 18, it can be seen that the Algorithm 2 not only successfully removes the MPC but also the strong clutter residual signals. The clutter components may not be strong enough for all the radar sensors simultaneously, so Algorithm 2 effectively removes the clutter noise from the radar signals. It may be worthy to note that according to Fig. 18 (Scenario 3), the two targets are separated by $1 \mathrm{~m}$ distance from radar $\mathrm{R} 2$ perspective. If a multipath threshold is applied as in [22] (with a value of $1 \mathrm{~m}$ ), then it would remove one target which results in miss detection. The problem with multipath removal based on a threshold value is that if the threshold is small, then it is not effective in removing the MPC, and if it is large, then it is hard to distinguish the adjacent human peaks. Hence, there is a tradeoff between efficiently removing MPC and distinguishing between adjacent human peaks. However, in the proposed MPC removal algorithm the distance between adjacent clusters is chosen as $20 \mathrm{~cm}$, which effectively allows distinguishing the adjacent human peaks while removing the MPC, as discussed in detail in Algorithm 2.

4) Positioning results: The radar sensors are deployed on the room walls, which confines the human motion, even though the radar detection range is larger than the size of room considered for experiments.

a) Positioning results of a single person: The 2D positioning was obtained by the trilateration technique. A median filter was also used to remove the outliers. The localization results for Scenario 1, where the person was standing in the range of radar sensors and Scenario 2, where the person is walking along the diagonal of the rectangular area, are presented in Fig. 19.

In Fig. 19a, the positioning result of a person standing at the center of the plane is given. The corresponding histogram shows that the density of positioning coordinates is centered at around the center of the plane. Although in the MPC result

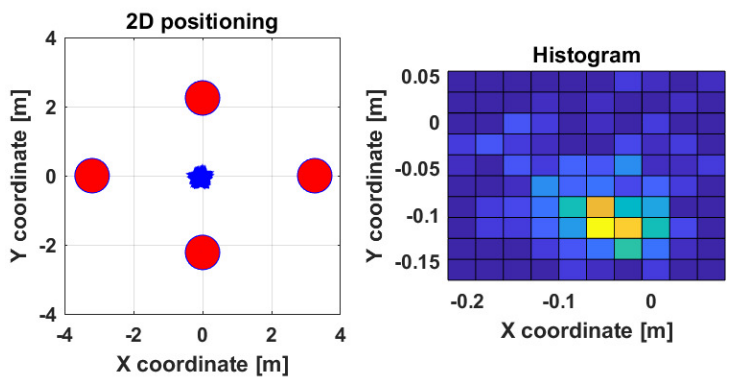

(a)

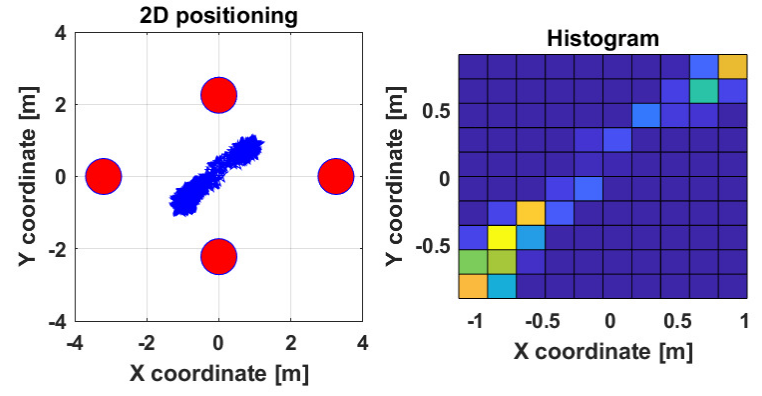

(b)

Fig. 19: Positioning result of a (a) standing person (b) walking person.
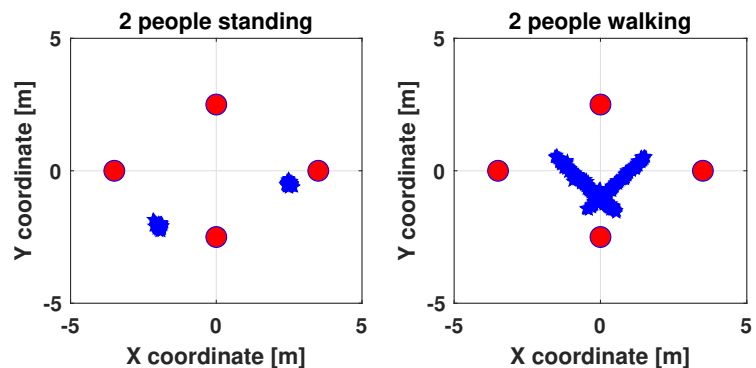

Fig. 20: Positioning results of two people (standing and walking).

for Scenario 2 in Fig. 16, there are some outliers, they are removed using a median filter before localization. Therefore, in Fig. 19b there are no outlier points. It can be noticed from Fig. 19b that the density of points is variable along the line of motion, as can be seen in the corresponding histogram. This effect is also visible in single radar ranging data shown in Fig. 18 (Scenario 2). The reasons of the missing data might be the heavy cluttered indoor environment and/or hardware problems such as antenna misalignment, as the antennas in the experiments have a beam width of 60 degrees. Missing data is currently an interesting area of research and advanced signal processing algorithms, such as those proposed in [52], may be used for predicting missing measurements.

b) Positioning results of two persons: In order to associate the positioning coordinates to the corresponding targets, JPDA algorithm is employed. The positioning results of the experiments of scenario 2 and scenario 3 are shown in Fig. 20. 

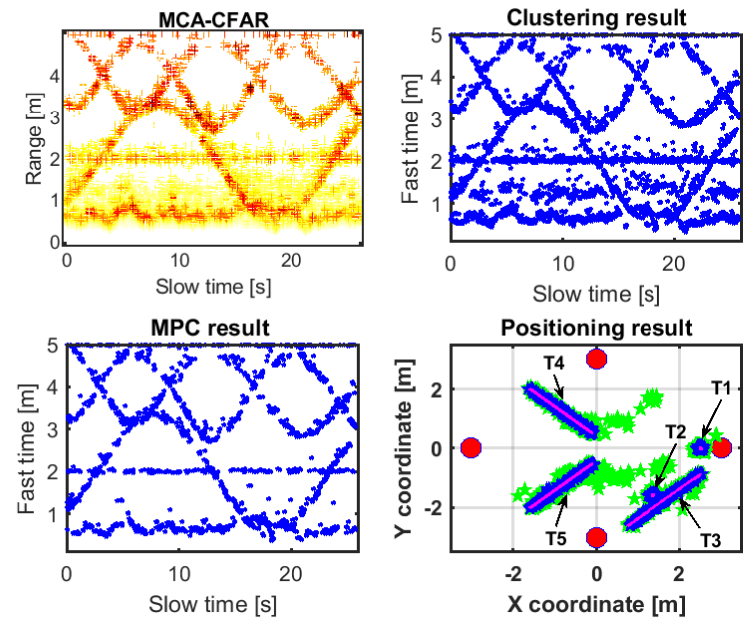

Fig. 21: Multi-target detection result

\section{Collision avoidance results (SET-UP B)}

For the experiments of collision avoidance, the experimental set-up shown in Fig. 15 was considered. Experiments were conducted with different people walking inside the given environment. The motion speed was around $1.5 \mathrm{~m} / \mathrm{s}$. The cart was also included in these experiments, where the targets were either moving or static.

1) Positioning results and discussion: After the detection and MPC removal stage, a clean signal is obtained as discussed in Section VI-B-3. A clustering algorithm is used to get the range of the targets, serving as input to the MPC reduction algorithm, which is followed by the trilateration and JPDA algorithms. The JPDA algorithm initializes, confirms, predicts and deletes tracks based on detections from multiple sensors. If a new detection does not belong to any existing tracks then it is assigned to a new track. The new track is tentative and if further detections are assigned to the tentative track then its status is changed from tentative to confirmed. The data association algorithm helps us in two ways. First, it gives us the status of each track and thus any collision can be avoided by using a distance threshold among tracks. Secondly, it is also useful for continuous classification of a target associated to a track. A CNN is applied to the target associated with each track as soon as the track is confirmed. Sometimes, the tracks come closer to each other and it is difficult to get the signatures of the targets due to mixing of the signals. But since the targets are classified continuously based on the tracks, therefore, in such scenarios where the signatures of objects get mixed, the history of the classification results of the tracks are used.

Fig. 21 shows the result provided by the proposed algorithms and the ground truth value for actual targets. A total of five targets are involved in this experiment: a static human (T1), a static machine (T2), and three moving humans (T3,T4 and T5). We chose the specific pattern of the motion instead of random motion so that the ground truth value can easily be determined. The blue line shows the tracking results of the proposed algorithm while the green points show the results of the trilateration algorithm with only 3 radar sensors. Ground truth is represented by magenta line. Although the green and
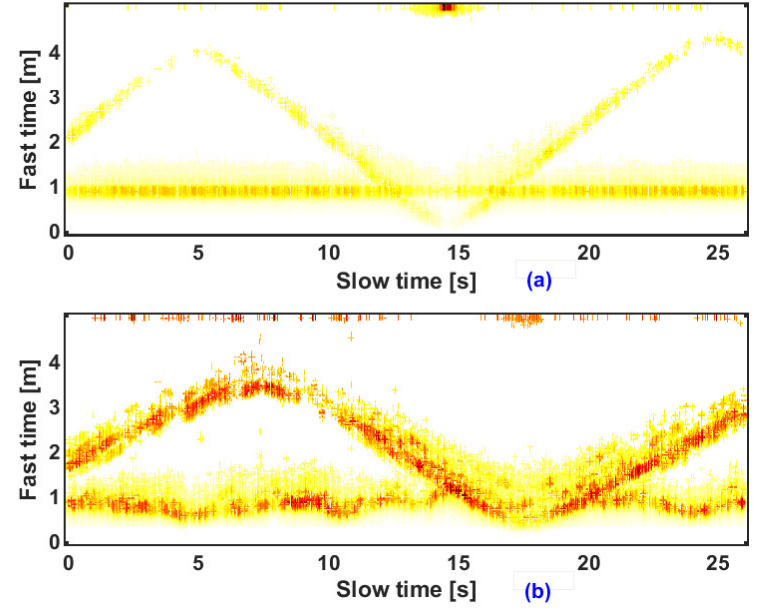

Fig. 22: Radar signatures for (a) scenario (ii) (b) scenario (iii). TABLE IV: CNN accuracy results.

\begin{tabular}{|c||c||c|}
\hline Actual class/Predicted class & Human & Cart \\
\hline Human & $91.7 \%$ & $8.3 \%$ \\
\hline Cart & $13.7 \%$ & $86.3 \%$ \\
\hline
\end{tabular}

blue lines overlaps for most of the time, however, there are many outlier green points as visible in the figure. Hence, it is clear that by using the 3 radar setup, there are many ghost targets detected by the conventional trilateration algorithm, while using the 4 radar setup helped to reduce the MPC and thus resulted in accurate positioning.

2) CNN classification results (SET-UP C): The experimental set-up considered in this part is shown in Fig. 15, section VI-A. Experiments were performed with a cart and four human subjects. The movement of the human and cart were performed with different speeds and orientations. The maximum walking speed was around $1.5 \mathrm{~m} / \mathrm{s}$. There were five different types of experiments performed to collect a set of data used for extracting images for training the $\mathrm{CNN}$ and testing the performance of the CNN. These experiments include (i) human standing at a fixed location. (ii) machine located at a fixed distance with the motion generated from the moving parts (iii) human body static and machine moving around (iv) human body walking and machine at a fixed location from the radar (v) both human and machine moving. For illustration purposes, the radar signatures for the scenarios (ii) and (iii) are shown in Fig. 22. It can be observed that in Fig. 22 a, the machine at a distance of around $1 \mathrm{~m}$ has a very smoother signature as compared to the human standing at around $1 \mathrm{~m}$ in Fig. 22 b. It is due to the fact that human body exhibits some natural motions due to the movement of different body organs even if the body is standing in front of the radar. The deep learning algorithm extracts such features automatically and use it for classification. The received radar data was transformed into images by employing the ROI extraction technique mentioned in section $\mathrm{V}$. The resulting images were then stored in a database for training the CNN. A total of 1500 images were stored for each class and randomly divided for training (80\%) and evaluation (20\%) purposes. The resulting confusion matrix is presented in Table IV. 
TABLE V: Execution time.

\begin{tabular}{|c||c||c|}
\hline System block & $\begin{array}{c}\text { Execution time } \\
\text { (milliseconds) }\end{array}$ & $\begin{array}{c}\text { Percentage of } \\
\text { total time [\%] }\end{array}$ \\
\hline $\begin{array}{c}\text { Clutter removal and } \\
\text { distance compensation }\end{array}$ & 1.2 & 3.2 \\
\hline Two stage MCA-CFAR & 25 & 67.3 \\
\hline Clustering algorithm & 5.4 & 14.5 \\
\hline MPC removal algorithm & 5.5 & 14.8 \\
\hline
\end{tabular}

\section{Execution time}

The PC used for signal processing has the following specifications: 4.0 GB RAM, Processor was Intel ${ }^{\circledR}$ Core TM i5-7200 CPU @ $2.50 \mathrm{GHz}$ and operating system was 64-bit Windows 10. The average processing time for each step of the system is shown in Table $\mathrm{V}$.

From Table V, it is clear that the execution time of each step is small enough for real time applications. The overall execution time for processing each backscattered signal in slow time is 37.1 millisecond. The image formation and CNN classification takes 93.8 milliseconds. Since the CNN makes a decision every 30 frames, it was not included along with the other algorithms, which are applied after each frame. As the slow time sampling frequency is 30 frames/seconds, the processing time of signal collected for 1 second is 1.1 second.

\section{CONCLUSION}

In this paper, multi-human/machine detection and classification for the purpose of collision avoidance in indoor environments has been discussed. A cluttered indoor environment has been considered for conducting real time experiments, which have resulted in strong clutter components as well as MPC. An MCA-CFAR algorithm has been proposed and evaluated to remove the spurious noise and clutter components from the backscattered signal. Another algorithm based on multiple radar set-up has been introduced and evaluated to remove the MPC from the received signals. A generic machine (cart with rotating fan), which can be both mobile and have moving parts, was also successfully distinguished from humans in this work using a deep learning CNN. Multiple scenarios have been considered for experimental verification of the algorithms, such as static and moving persons in indoor environment. In addition, time processing results have been included to demonstrate that the proposed algorithms are well suited for real life applications.

As future work, we plan to extend the proposed algorithms to the multi-humans localization and tracking using multiple radar sensors and to consider the detailed performance analysis of the detection system with respect to the multiple adjustable parameters. Further, more radar set-ups may be included for scaling the system for larger areas inside more realistic smart factory environments.

\section{ACKNOWLEDGMENT}

This research was supported by the MSCA-IF grant for the H2020 UWB-IODA project, the IBNM (Brest Institute of Computer Science and Mathematics) and the Natural Sciences and Engineering Research Council of Canada (NSERC) through its Discovery program.

\section{REFERENCES}

[1] G. Monaci and A. V. Pandharipande, "Passive infrared sensor system for position detection." U.S. Patent No. 10,209,124., 2019.

[2] J. Zhao, H. Xu, H. Liu, J. Wu, Y. Zheng, and D. Wu, "Detection and tracking of pedestrians and vehicles using roadside LiDAR sensors," Transportation Research Part C: Emerging Technologies, vol. 100, pp. 68-87, 2019. [Online]. Available: https://www.sciencedirect.com/science/article/pii/S0968090X19300282

[3] X. Guo, L. Bo, S. Cong, L. Hongbo, C. Yingying, and C. C. Mooi, "WiFi-enabled smart human dynamics monitoring." in Proceedings of the 15th ACM Conference on Embedded Network Sensor Systems, 2017, pp. 1-13.

[4] S. Ren, K. He, R. Girshick, and J. Sun, "Faster R-CNN: towards realtime object detection with region proposal networks." IEEE Transactions on Pattern Analysis and Machine Intelligence, vol. 39, no. 6, pp. 11371149, 2016.

[5] K. Bregar, H. Andrej, and M. Mohorcic, "NLOS channel detection with multilayer perceptron in low-rate personal area networks for indoor localization accuracy improvement." in Proceedings of the 8th Jožef Stefan International Postgraduate School Students' Conference, Ljubljana, Slovenia, vol. 31, 2016.

[6] T. Wei and Z. Xinyu, "mtrack: High-precision passive tracking using millimeter wave radios," in Proceedings of the 21 st Annual International Conference on Mobile Computing and Networking, 2015, pp. 117-129.

[7] J. Palacios, G. Bielsa, P. Casari, and J. Widmer., "Single-and multipleaccess point indoor localization for millimeter-wave networks." IEEE Transactions on Wireless Communications, vol. 18, no. 3, pp. 19271942, 2019

[8] X. Huang, C. Hasnain, T. Abin, and J. K. Tsoi., "Single-and multipleaccess point indoor localization for millimeter-wave networks." Journal of Sensors, p. 1, 2021.

[9] X. Yang, W. Yin, L. Li, and L. Zhang, "Dense people counting using IRUWB radar with a hybrid feature extraction method," IEEE Geoscience and Remote Sensing Letters, vol. 16, no. 1, pp. 30-34, 2019.

[10] S. Chang, T.-S. Chu, J. Roderick, C. Du, T. Mercer, J. W. Burdick, and H. Hashemi, "UWB human detection radar system: A RF CMOS chip and algorithm integrated sensor," in 2011 IEEE International Conference on Ultra-Wideband (ICUWB), 2011, pp. 355-359.

[11] X. Yang, W. Yin, and L. Zhang, "People counting based on CNN using IR-UWB radar," in 2017 IEEE/CIC International Conference on Communications in China (ICCC), 2017, pp. 1-5.

[12] S. Bottigliero, D. Milanesio, M. Saccani, and R. Maggiora, "A lowcost indoor real-time locating system based on TDOA estimation of UWB pulse sequences," IEEE Transactions on Instrumentation and Measurement, vol. 70, pp. 1-11, 2021.

[13] F. Khan, G. Asim, N. Khan, and S. H. Cho, "An overview of signal processing techniques for remote health monitoring using impulse radio UWB transceiver." Sensors, vol. 20, no. 9, p. 2479, 2020.

[14] L. Barbieri, M. Brambilla, A. Trabattoni, S. Mervic, and M. Nicoli, "UWB localization in a smart factory: Augmentation methods and experimental assessment," IEEE Transactions on Instrumentation and Measurement, vol. 70, pp. 1-18, 2021.

[15] W. Suski, S. Banerjee, and A. Hoover, "Using a map of measurement noise to improve UWB indoor position tracking," IEEE Transactions on Instrumentation and Measurement, vol. 62, no. 8, pp. 2228-2236, 2013.

[16] S. Chang, R. Sharan, M. Wolf, N. Mitsumoto, and J. W. Burdick, "UWB radar-based human target tracking," in 2009 IEEE Radar Conference, 2009, pp. 1-6.

[17] J. R. Fernandes and D. Wentzloff, "Recent advances in IR-UWB transceivers: An overview," in Proceedings of 2010 IEEE International Symposium on Circuits and Systems, 2010, pp. 3284-3287.

[18] M. Ghavami, L. Michael, and R. Kohno, Ultra Wideband Signals and Systems Communication Engineering. John Wiley '\&' Sons, Ltd, 2007, pp. 1-8.

[19] J. Maitre, K. Bouchard, C. Bertuglia, and S. Gaboury, "Recognizing activities of daily living from UWB radars and deep learning," Expert Systems with Applications X, vol. 164, 092020.

[20] J. Fontaine, M. Ridolfi, B. Van Herbruggen, A. Shahid, and E. De Poorter, "Edge inference for UWB ranging error correction using autoencoders," IEEE Access, vol. 8, pp. 139 143-139 155, 2020.

[21] J.-H. Choi, J.-E. Kim, N.-H. Jeong, K.-T. Kim, and S.-H. Jin, "Accurate people counting based on radar: Deep learning approach." 2020 IEEE Radar Conference (RadarConf20), pp. 1-5, 2020.

[22] J.-H. Choi, J.-E. Kim, and K.-T. Kim, "People counting using IR-UWB radar sensor in a wide area," IEEE Internet of Things Journal, vol. 8 , no. 7, pp. 5806-5821, 2021. 
[23] J. H. Choi, J. E. Kim, and K. T. Kim, "Learning-based people counting system using an ir-uwb radar sensor." The Journal of Korean Institute of Electromagnetic Engineering and Science, vol. 30, no. 1, pp. 28-37, 2019.

[24] J. W. Choi, D. H. Yim, and S. H. Cho, "People counting based on an IR-UWB radar sensor," IEEE Sensors Journal, vol. 17, no. 17, pp. 5717-5727, 2017.

[25] S. Sun, J. Hu, J. Li, R. Liu, M. Shu, and Y. Yang, "An INS-UWB based collision avoidance system for AGV," Algorithms, vol. 12, p. 40, 02 2019.

[26] A. Alarifi, A. Al-Salman, M. Alsaleh, A. Alnafessah, S. Alhadhrami, M. Al-Ammar, and H. Al-Khalifa, "Ultra wideband indoor positioning technologies: Analysis and recent advances," Sensors, vol. 16, pp. 1-36, 052016.

[27] X. Li, D. Li, J. Wan, A. Vasilakos, C. Lai, and S. Wang, "A review of industrial wireless networks in the context of Industry 4.0," Wireless Networks, vol. 23, pp. 23-41, 2017.

[28] M. Saputra and P. Santosa, "Obstacle avoidance for visually impaired using auto-adaptive thresholding on kinect's depth image." IEEE 11th Intl Conf on Ubiquitous Intelligence and Computing and 2014 IEEE 11th Intl Conf on Autonomic and Trusted Computing and 2014 IEEE 14th Intl Conf on Scalable Computing and Communications and Its Associated Workshops, pp. 337-342, 2014

[29] J. Han, K. Cho, Y., J. J., Kim, N. Son, and S. Kim, "Autonomous collision detection and avoidance for ARAGON USV: Development and field tests." Journal of Field Robotics, vol. 37, no. 6, pp. 987-1002, 2020.

[30] B. Silva and G. P. Hancke, "IR-UWB-based non-line-of-sight identification in harsh environments: Principles and challenges," IEEE Transactions on Industrial Informatics, vol. 12, no. 3, pp. 1188-1195, 2016.

[31] A. H. Muqaibel, M. A. Landolsi, and M. N. Mahmood, "Practical evaluation of NLOS/LOS parametric classification in UWB channels," in 2013 1st International Conference on Communications, Signal Processing, and their Applications (ICCSPA), 2013, pp. 1-6.

[32] J. Khodjaev, Y. Park, and A. Malik, "Survey of NLOS identification and error mitigation problems in UWB-based positioning algorithms for dense environments," Annales des Télécommunications, vol. 65, pp. 301-311, 062010.

[33] V. Barral, C. Escudero, J. García-Naya, and R. Maneiro-Catoira, "NLOS identification and mitigation using low-cost UWB devices," Sensors, vol. 19, p. 3464, 082019.

[34] H. Wymeersch, S. Marano, W. M. Gifford, and M. Z. Win, "A machine learning approach to ranging error mitigation for UWB localization," IEEE Transactions on Communications, vol. 60, no. 6, pp. 1719-1728, 2012.

[35] S. Maranò, W. M. Gifford, H. Wymeersch, and M. Z. Win, "NLOS identification and mitigation for localization based on UWB experimental data," IEEE Journal on Selected Areas in Communications, vol. 28, no. 7, pp. 1026-1035, 2010.

[36] C. Jiang, J. Shen, S. Chen, Y. Chen, D. Liu, and Y. Bo, "UWB NLOS/LOS classification using deep learning method," IEEE Coтmunications Letters, vol. 24, no. 10, pp. 2226-2230, 2020.

[37] J. Shen and F. M. Andreas, "Estimation of multiple target location in multi-path wireless systems." in 2013 IEEE Global Communications Conference (GLOBECOM), 2013, pp. 4319-4324.

[38] J. Shen and A. F. Molisch, "Estimating multiple target locations in multipath environments," IEEE Transactions on Wireless Communications, vol. 13, no. 8, pp. 4547-4559, 2014.

[39] Y. Bar-Shalom, F. Daum, and J. Huang, "The probabilistic data association filter," EEE Control Systems Magazine, vol. 29, no. 6, pp. 82-100, 2009.

[40] A. D. Pham and H. J. Ahn, "High precision reducers for industrial robots driving 4th industrial revolution: state of arts, analysis, design, performance evaluation and perspective." International journal of precision engineering and manufacturing-green technology, vol. 5, no. 4, pp. 519533, 2018.

[41] D. S. Van, H. J. Degezelle, P. De, J. Schutter, and R. J. Belmans, "Servo motor selection criterion for mechatronic applications." IEEE/ASME Transactions on mechatronics, vol. 3, no. 1, pp. 43-50, 1998.

[42] Y. Ma, F. Liang, P. Wang, H. Lv, X. Yu, Y. Zhang, and J. Wang, "An accurate method to distinguish between stationary human and dog targets under through-wall condition using UWB radar." Remote Sensing, vol. 11 , no. 21 , p. $2571,2019$.

[43] Q. Wu, T. Gao, Z. Lai, and D. Li, "Hybrid SVM-CNN classification technique for human-vehicle targets in an automotive LFMCW radar." Sensors, vol. 20, no. 12, p. 3504, 2020.

[44] F. Khan, S. Leem, and S. H. Cho, "Hand-based gesture recognition for vehicular applications using IR-UWB radar," Sensors, vol. 17, 042017.
[45] J. W. Choi, S. S. Nam, and S. H. Cho, "Multi-human detection algorithm based on an impulse radio ultra-wideband radar system," IEEE Access, vol. 4, pp. 10300-10309, 2016.

[46] B. Mahafza, "Radar systems analysis and design using MATLAB." Chapman and Hall/CRC, 2005.

[47] G. J. Hahn and S. S. Shapiro, Statistical Models in Engineering. New York: Wiley Classics Library, 1994.

[48] T. Black, P. Pathirana, and S. Nahavandi, "Position estimation and tracking of an autonomous mobile sensor using received signal strength." in 2008 International Conference on Intelligent Sensors, Sensor Networks and Information Processing, 2008, pp. 19-24.

[49] N. Andersen, G. Kristian, A. Jørgen, S. B. Michaelsen, H. A. Hjortland, R. K. Mats, T. S. Lande, and D. T. Wisland., "A 118-mw pulse-based radar SoC in 55-nm CMOS for non-contact human vital signs detection." IEEE Journal of Solid-State Circuits, vol. 52, no. 12, pp. 3421-3433, 2017.

[50] X4-datasheet. [Online]. Available: https://novelda.com/content/wpcontent/uploads/2021/01/NOVELDA-x4-datasheet-revF.pdf

[51] N. Van Han and J.-Y. Pyun, "Location detection and tracking of moving targets by a 2d IR-UWB radar system," Sensors (Basel, Switzerland), vol. 15, pp. 6740-62, 032015.

[52] N. Khan, "Linear prediction approaches to compensation of missing measurements in Kalman filtering," Ph.D. dissertation, University of Leicester, 112011. 